\title{
LOS DOBLETES ETIMOLÓGICOS EN ESPAÑOL: DEL CAMBIO FONÉTICO AL PROBLEMA CONCEPTUAL
}

\author{
The double etymological words in Spanish: from phonetic change to a \\ conceptual problem
}

Gilberto Castro Delgado ${ }^{1}$

\begin{abstract}
RESUMEN
En la siguiente ponencia se tratarán algunos problemas que se dan en el uso de los dobletes etimológicos del español, en el sentido de que con su uso se hace una clara diferenciación social de los hablantes de la lengua. Conviene, por lo tanto, señalar algunos principios conceptuales para la interpretación contextual, histórica y lexicogénica de las palabras que forman dobletes etimológicos: metáfora, vaguedad, prototipos, aires de familia, categorización, etc. Estos principios pueden brindar un apoyo muy importante en la concepción de ideas que lleven a la correcta asociación de las palabras dentro de una red conceptual.
\end{abstract}

Palabras clave: vaguedad, prototipo, categorización, metáfora, lexicogénesis.

\begin{abstract}
The next pages are about some problems that result from the use of the double etymological words in Spanish. We mean that, because of this, there is a social differentiation between speakers of the Spanish language. Then, it's necessary to point up some conceptual items that make comprehensive the contextual, historical and lexicogenic interpretation of the double etymological Spanish words: metaphor, vagueness, prototypes, word resemblance, categorization, etc. These items can support the importance of the ideas in a correct practical network within a conceptual network.
\end{abstract}

Key Words: vagueness, prototype, categorization, metaphor, lexicogenesis.

\section{Introducción}

El objetivo de las siguientes líneas consiste en determinar algunas diferencias, dentro de un orden conceptual, entre las formas del léxico latino heredadas en el español a través de su evolución histórica y las formas léxicas que conforman otra extensión del léxico latino en el español, las que han penetrado por la vía académica.

\footnotetext{
${ }^{1}$ Universidad de Costa Rica. Profesor de la Escuela de Filología, Lingüística y Literatura. Costa Rica. Correo: gilberto.castro@ucr.ac.cr
}

Recepción: 22/6/17 Aceptación: 5/10/17. 
Hay que tener en cuenta que, históricamente, las lenguas se diferencian en distintas etapas de su desarrollo, de modo que las palabras y las frases están sometidas a una discriminación radical por los efectos de las clases sociales y entornos culturales entre los hablantes, lo cual se ve como una presión al cambio inevitable en el camino evolutivo de la lengua, especialmente los cambios gramaticales (fonética, morfología y sintaxis). Dicha discriminación debe abordarse como un mecanismo de pervivencia de los hechos de cultura dentro de la lengua, a pesar de las manifestaciones de los cambios fonéticos, principalmente, y de nuevas manifestaciones culturales que se generan en una nueva etapa de la lengua.

A lo anterior hay que agregar el hecho de que los elementos evolutivos están en cierto modo prefigurados y determinados, como mencionan Herman (2001) y Quirós (2004), por la estructura del latín, lo que determina tipos de declinación asimétricas, funciones variadas de los distintos casos, etc. Y también, dice Herman (2001), existe la posibilidad de que se diera un contacto con elementos importados, como los esclavos que aprendieron el latín de manera oral y sin una adecuada sistematización didáctica; además del contacto en las provincias.

Por otro lado, es claramente observable que el léxico del latín vulgar estaba bastante diferenciado del vocabulario del latín culto. Léxico, fonética y dialectalismo marcaron una vía de solución hacia la proyección de resultados satisfactorios para la constitución de la nueva lengua. También hay que destacar los resultados alcanzados en los períodos de contacto de las lenguas neolatinas con la cultura clásica.

\section{La categoría léxico}

"Words glisten. Words irradiate exquisite splendour. Words carry magic and keep us spell-bound (...).Words are like glamorous bricks that constitute the fabric of any language (...).Words are like roses that make the environment fragrant", asserts the writer of a textbook urging people to improve their vocabulary (Jean Aitchison, 1987, p. 3).

En la cita anterior es notoria la afirmación de que las palabras contienen todo un esplendor y toda una magia en su uso en la lengua. En sí, es también una invitación al correcto uso del léxico de una lengua dentro de su amplia y vasta red de significados y formas afines 
para el mejoramiento de la competencia léxica de un hablante. Esta idea nos acerca a la concepción, según Lyons (1995), de que el léxico es un conjunto o red de lexemas de una lengua almacenada en el cerebro de los hablantes competentes, el cual contiene información lingüística para cada lexema requerida para la producción e interpretación de los enunciados en la lengua.

Ser competente en una lengua implica tener un conocimiento bastante amplio de significados léxicos y gramaticales de los lexemas, como los denomina Lyons (1995). A eso se refieren las frases que indican que las palabras brillan, las palabras son radiantes de un esplendor cultural de una época, de movimientos culturales y de razonamiento gramatical. Un buen uso del léxico proyecta una dimensión extraordinaria de la cultura, aunque también las palabras, en tanto que conforman redes conceptuales, ponen en peligro la pervivencia léxica.

Estas ideas nos recuerdan que no deben abandonarse los argumentos por tratar de ver en los aspectos culturales el uso de la lengua. La estructura del lenguaje está motivada por la percepción humana del mundo, menciona Moure (1996); hay un conocimiento linguiístico semántico- que deriva del conocimiento cultural, y este, dice Moure (1996), es un subconjunto de aquel, en cierta forma, esbozado por unos lindes difusos. La semántica va ligada a la pragmática.

El léxico o vocabulario es donde mejor se manifiesta la pervivencia y renovación de una lengua, afirma Väänänen (1975), como también muchos otros estudiosos de la lexicología y la lexicografía. El vocabulario de una lengua es el aspecto gramatical más dinámico, es decir, los elementos léxicos están en constante cambio; estos se renuevan o se pierden. Mediante la renovación se puede implicar la pérdida de elementos léxicos obsoletos, o puede producir también un cambio de significado en la palabra, la cual podría más tarde tener su propia evolución fonética.

Es cierto, las palabras envejecen y caen en desuso, o se conservan en estratos sociales determinados (religión, diplomacia, agricultura, legislación, etc.) y en regionalismos; por otra parte, hay palabras que penetran directamente en ámbitos culturales establecidos o en la tecnología. Menciona Väänänen (1975) el término terapéutica lingüística, acuñado por J. 
Guillieron, para indicar que las palabras usadas que no forman imagen dan marcha atrás y hasta zozobran en beneficio de otras expresiones más vigorosas.

Las palabras forman una imagen. Parece que Väänänen ya visualizaba, a partir del vocabulario latino vulgar, la idea de que las palabras no son la suma de rasgos discretos, sino que constituyen unidades simbólicas dotadas de una irradiación conceptual que las hacen circunscribirse en el mundo real bien organizado y provisto de una amplia gama de información cognitiva. La irradiación enciclopédica de una imagen se vincula a la concepción del lenguaje como un edificio construido con ladrillos que se conectan como una verdadera red conceptual, tal y como dice la cita anterior de Aitchinson.

\section{Dobletes etimológicos}

Se sabe que el vocabulario español está constituido en su mayor parte por palabras heredadas del latín, por la vía del latín del pueblo, el latín vulgar; es decir, palabras que se han mantenido en el uso de los hablantes por la vía oral y que manifiestan una diversidad de cambios fonéticos que las hacen muchas veces irreconocibles con respecto a su modelo original. Son las llamadas voces populares. Hay también semicultismos, palabras que no han seguido el curso evolutivo tradicional y se encuentran más cercanas al modelo latino originario; solo sufrieron algunas modificaciones indispensables para adaptarse a la pronunciación, menciona Pino (2009). Por otra parte, a lo largo de toda su historia el español ha incorporado en su léxico, a través de la escritura, ${ }^{2}$ un gran número de palabras latinas, bien directamente de esta lengua, bien a través de otra lengua moderna; ${ }^{3}$ son los llamados 'cultismos' o 'latinismos' que han servido para enriquecer el vocabulario culto, las lenguas de especialidad y los tecnicismos.

Estas tres vías -voces populares, semicultismos y cultismos-, por las que el léxico latino ha evolucionado en español, quedan a veces reflejadas en distintas derivaciones de un

\footnotetext{
${ }^{2}$ Hay que recordar los movimientos culturales en los que los países europeos acogieron el inmenso legado de la tradición grecolatina, como el humanismo y, posteriormente, la ilustración; momentos en que las lenguas se nutren de particularidades gramaticales, léxicas y literarias, dándose principalmente la acogida de latinismos en las lenguas modernas.

${ }^{3}$ Préstamos del francés, del italiano, del occitano, etc.
} 
mismo vocablo español; por ejemplo regula ha dado en español reja (voz popular), regla (semicultismo) y regular (cultismo).

Quirós (2000) define los dobletes etimológicos de la siguiente manera:

Los dobletes etimológicos son dobles variaciones, léxica y semántica, de una palabra latina, debido a una procedencia románica en series paralelas: un cultismo y una voz popular, un cultismo y un semicultismo o un semicultismo y una voz popular, en donde tanto el cultismo como el semicultismo son extraídos de la tradición culta del latín, y la voz popular es una herencia o evolución normal de la palabra latina vulgar (p. 115).

Se puede entender entonces que los dobletes son el emparejamiento de palabras procedentes de un mismo étimo, pero que se diferencian en que uno es casi un calco hecho sobre la palabra de la lengua madre con variaciones fonéticas mínimas y el otro se analiza dentro de las leyes fonéticas regulares de una lengua. Este emparejamiento es lo que causa un problema conceptual, debido a que los hablantes o usuarios de la lengua deben reconocer en las formas alternantes de una raíz los aspectos semánticos o gramaticales que esta encierra.

Algunos ejemplos de dobletes etimológicos en español son:

Tabla 1. Dobletes etimológicos

\begin{tabular}{lll}
\hline LATÍN & VOZ POPULAR & $\begin{array}{l}\text { VOZ CULTA O } \\
\text { SEMICULTA }\end{array}$ \\
\hline hominem $^{4}$ & $\begin{array}{l}\text { hombre } \\
\text { hombrada (hacer la }\end{array}$ & $\begin{array}{l}\text { homínido-homicidio } \\
\text { (humano-humilde) }\end{array}$ \\
\hline feminam & hembra & femenino-feminista \\
\hline operare & obrar \\
obrar(se) & reja & operativo-operar (ópera) \\
\hline regulam & frío & $\begin{array}{l}\text { regla (venírsele la regla)- } \\
\text { regular }\end{array}$ \\
\hline frigidum & fruto & frigidez \\
\hline fructum & mancha & infructuoso \\
\hline maculam & habla & inmaculada \\
\hline fabula & fábula-fabuloso \\
\hline
\end{tabular}

${ }^{4}$ Estas formas terminadas en $\mathrm{m}$ - corresponden al caso acusativo singular del cual proceden, en su mayoría, las palabras del español. 


\begin{tabular}{|c|c|c|}
\hline computare & contar & computadora-computar \\
\hline ferrum & $\begin{array}{l}\text { hierro-herrero } \\
\text { herrumbre }\end{array}$ & ferretería \\
\hline clavem & llave & clave \\
\hline alienum & ajeno-enajenación & $\begin{array}{l}\text { alienación-alienígena- } \\
\text { inalienable }\end{array}$ \\
\hline limpidus & limpio & límpido \\
\hline mensem & mes & mensual-menstruación \\
\hline annum & año & anual-bienal \\
\hline capitalem & caudal & capital-capitolio \\
\hline limitem & linde-lindero & límite-limitar \\
\hline radium & rayo & radial-radio \\
\hline \multirow{2}{*}{$\begin{array}{l}\text { rupturam } \\
\text { rotellam } \\
\text { rotulam }\end{array}$} & rotura & ruptura \\
\hline & rodilla & rótula \\
\hline collocare & colgar & colocar \\
\hline pensare & pesar & pensar-pensamiento \\
\hline nutriciam & nodriza & nutricia \\
\hline vaginam & vaina & vaginal \\
\hline strictum & estrecho & estricto \\
\hline sextam & siesta & sexteto \\
\hline seminarium & semillero & seminarista \\
\hline digitalem & dedal & digital-digitalizar-dígito \\
\hline directam & derecha & directa \\
\hline legalem & leal & legalem \\
\hline colectam & cosecha & colecta-colectivo \\
\hline diem (diam) & día & diario- a diario-meridiano \\
\hline noctem & noche & nocturno \\
\hline lactem & leche-lechero-lechón & lácteo-lactosa-lactancia \\
\hline lacrimam & lágrima & lacrimógeno \\
\hline iudicem & juez & judicial \\
\hline laborare & $\begin{array}{l}\text { labrar-labranza } \\
\text { labriego }\end{array}$ & laboral-laboratorio \\
\hline pedem & pie & pedal-pedestre \\
\hline oculum & ojo & oculista-ocular \\
\hline auriculam & oreja & auricular (auditivo-audio) \\
\hline honorare & honrar-honroso & $\begin{array}{l}\text { honorífico-honorable } \\
\text { (honesto) }\end{array}$ \\
\hline $\operatorname{cor}(d)$ & corazón $^{5}$ & cordial \\
\hline fabricare & fraguar & fabricar \\
\hline recitare & rezar & recitar \\
\hline fastidium & hastío & fastidio \\
\hline
\end{tabular}

\footnotetext{
${ }^{5}$ En francés coeur; en italiano cuore.
} 
Esto es solo una reducida muestra del vocabulario español que contiene dobletes etimológicos desde el latín, pero que refleja una clara diferencia de las formas, aunque en algunos ejemplos se reconocen con mayor facilidad las alternancias de las formas. Podrá notarse que algunas palabras, debido a su evolución fonética, llegan a perder todo su carácter semasiológico y son irreconocibles sin una detallada atención. En muchos casos, hay un distanciamiento conceptual de las palabras. Podrán notarse algunos cambios según las leyes fonéticas del español, tales como la diptongación, la sonorización, síncopas, cambio de flatina inicial en h-, epéntesis, etc.

Compárense las palabras fabricar y fraguar. Sin duda, son dos lexemas completamente distintos en español, aunque provienen de la misma palabra latina, fabricare, derivado de fabrica 6 (arte, oficio; taller) y este derivado, a la vez, de faber (artesano). Definitivamente, los dos lexemas marcan un distanciamiento conceptual en su significación en español.

Como menciona Lara (2006), resulta difícil resolver el problema conceptual de los dobletes etimológicos, debido a que los cambios fonéticos producen formas funcionales de la palabra que no siempre resulta sencillo reconocer. Si se quiere apreciar esta alternancia de raíces o morfos, por usar el término de Mattews (1980), lo conveniente sería seguir un curso a nivel universitario o autodidacta, de fonética o fonología histórica del español, o de latín, para saber en qué consiste la abstracción de esa unidad subyacente.

\subsection{Motivación}

Las teorías lingüísticas modernas, como la gramática cognitiva, aplican el concepto motivación para dejar entrever una adyacencia semántica de los lexemas. Este es un concepto filosófico que se ha aplicado desde las ideas de Platón, quien en su tratado Cratilo argumentaba que las palabras debían tener su motivación en una completa comprensión de la escena de lo que significan, como indica Lara (2006), marcándose una verdadera relación

\footnotetext{
${ }^{6}$ Había expresiones referidas a fabrica, como aeraria fabrica, para indicar el trabajo propio de quienes trabajaban el metal. Por elipsis (ferraria fabrica) se creó el verbo fabricare > fraguar y con esto el término quedó referido exclusivamente a estos artesanos.
} 
entre la palabra y la cosa significada. Dentro de esta apreciación, Lara hace entender que deben existir relaciones formales o culturales entre vocablos.

La motivación está presente en el signo lingüístico, afirman Panther y Radden (2011), en el entendido de que este se constituye por el par forma-contenido, y puede alcanzar límites de significación en los distintos niveles gramaticales; en el caso del léxico, también en la composición de palabras. Así que el concepto de red conceptual abarca incluso los aspectos morfológicos, en los que la motivación se guía por factores independientes a la lengua, en especial el factor cognitivo.

De modo que existen distintos factores que determinan el uso de las unidades léxicas a partir de los conceptos de orden conceptual. Algunos hechos están ligados al carácter motivacional en las prácticas lingüísticas, como los propuestos por Gleitman y Landau (1996): la naturaleza del léxico mental, el descubrimiento del comportamiento de las unidades léxicas, un ordenamiento categorial del mundo que se vincula a hechos de categorización, de análisis verbal y de confrontación de estas en el lenguaje.

\subsection{Alomorfia}

Bybee (1985) y Halliday y Yallop (2007) concuerdan en que las palabras deben tener una similitud semántica y fonológica para tener correspondencia en una misma unidad léxica. Dicha correspondencia obedece a un principio de conexión léxica, la cual puede ser de orden semántico: fabul- /habl-, ferr-/hierr-, noct-/noch-, etc., o de orden morfológico como los dobletes etimológicos ejemplificados anteriormente, que dan una muestra de que existe tal similitud y los valores de unificación de ellos están representados por una alternancia de la raíz: femin-/hembr-, mens-/mes, homin-/hombr-, frigid-/fri, alien-/ajen-, etc. Si no se da tal similitud, entonces debe haber una conexión léxica con una base contextual.

De esto se deduce que la relación entre lexemas se hace más fuerte si hay un paralelo de las conexiones semánticas y fonológicas; además, se deduce que las palabras guardan una distancia en un campo conceptual, lo que hace que se distancien más aún en el tiempo y en el espacio. Bybee (1985) utiliza el término fortaleza léxica ${ }^{7}$ para argumentar que las palabras

\footnotetext{
${ }^{7}$ En inglés, lexical strength.
} 
toman importancia o se destacan cuando, después de producirlas y escucharlas, dejan una huella en el léxico. Los dobletes etimológicos cumplen con la función de proyectar las palabras en dos ámbitos de uso muy particulares, el uso popular y el uso culto, pudiendo también fortalecerse un campo intermedio gracias al academicismo de la enseñanza.

Por otra parte, ante estos casos de alomorfia (Bybee, 1985; Varela, 2005) -regul/regl-, limit-/lind-, seminar-/semiller-, clav-/llav-, etc.- el carácter funcional de los morfos debe distinguirse en la conjunción de presupuestos teóricos en morfología léxica. Halliday y Yallop (2007) analizan el léxico de una lengua mediante constructos léxicos en los que debe prevalecer un principio léxico-gramatical, ${ }^{8}$ el cual se entiende como una red de opciones a través de las cuales hay que localizarlos en el léxico mental del usuario: esto podría implicar el factor gramatical también inherente en la mente. Dentro de una red conceptual y en su carácter sistemático, el español presenta otras formas morfológicas alternantes, por ejemplo, en su sistema de conjugación, que motivan a que el usuario no tenga que hacer un gran esfuerzo por hacer inteligible su discurso: yo juego/nosotros jugamos; yo tengo/él tiene, etc.

En resumen, los lexemas, como dice Lyons, llegan a institucionalizarse en el sentido de que adquieren un significado más o menos especializado, gracias a la contextualización morfológica que deriva de las reglas morfológicas y de aspectos pragmáticos como la frecuencia y ámbito de uso. Hay una petrificación léxica, dice Lyons, debido a que las expresiones y palabras se construyen regularmente con un uso determinado por una ocasión de enunciación y están a disposición del usuario en forma de unidad rígida incorporable a futuras enunciaciones.

\subsection{Carácter conceptual del léxico}

De lo dicho hasta ahora, se puede mencionar que los conceptos, las palabras, no existen en el vacío, sino en relación con otros ámbitos del conocimiento más general, como dicen Cuenca y Hilferty (2007). De ahí que sea necesario hablar de dominios cognitivos para involucrar el léxico dentro de estos. Los dominios cognitivos son representaciones mentales

\footnotetext{
${ }^{8}$ En inglés, lexicogrammar.
} 
de cómo se organiza el mundo e incluyen una amplia gama de información, según Cuenca y Hilferty (2007), quienes afirman que el plano semántico es inherentemente enciclopédico.

Considerar el aspecto enciclopédico del léxico (y de la gramática en general) permite asociar con mayor fuerza los aspectos denotativos (estrictamente léxicos) con los connotativos (lo contextual, lo pragmático). Por lo tanto, debe considerarse que las palabras no son la suma de rasgos componenciales discretos, mencionan Cuenca y Hilferty; por el contrario, el carácter enciclopédico o de conocimiento general se entiende mejor cuando la gramática se analiza en dimensiones de categorías radiales y aires de familia, en el entendido de que los lexemas, a la vez, constituyen unidades simbólicas que pueden llegar a convertirse en unidades simbólicas complejas, como sucede en la gramática cognitiva. El conocimiento lingüístico y el conocimiento de mundo van de la mano.

De los conceptos adquiridos en la gramática cognitiva, dos son importantes para elaborar la dimensión cognoscitiva o enciclopédica en los dobletes etimológicos: los conceptos de perfil y base. Según Cuenca y Hilferty (2007), el perfil y la base son complementarios uno de otro; sin uno, el otro pierde su dimensión conceptual. Esto se ejemplifica bien en el modelo del triángulo. El concepto matemático hipotenusa es inherente al concepto triángulo y solo puede comprenderse si se ubica en adyacencia al resto de las líneas y ángulos pertinentes.

Tomando un lexema de los dobletes etimológicos anteriores, se puede ilustrar esta conceptualización. Por ejemplo, la palabra española siesta que proviene del latín sexta; en un diagrama geométrico, esta palabra quedaría representada así:

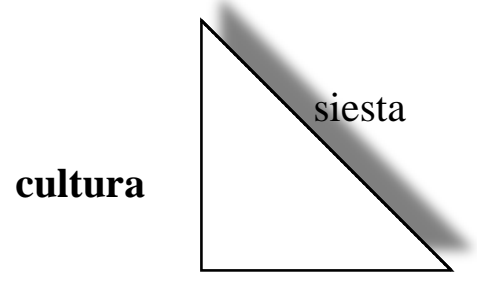

romana 
En español, se denomina siesta a un espacio de tiempo que se ubica después de la hora de almuerzo al mediodía, en correspondencia con un aspecto cultural, consuetudinario de la tradición latina. Después del almuerzo, los romanos acostumbraban tomarse un descanso. La hora del almuerzo era en la sexta hora ${ }^{9}$ y, por elipsis, se llamó a este intervalo sexta que, con los cambios fonéticos regulares del español, tenemos siesta. Si se analiza esta conceptualización, se verá que este lexema se ajusta a hechos de cultura y corresponde a otra idea cognitiva dentro de modelos cognitivos idealizados; aquí se está ante un caso de institucionalización semántica que desemboca en principios de prototipicidad y de categorización radial: no resulta difícil asociar sexta con un modelo cognitivo idealizado de la salud (descanso) y de lo consuetudinario (en cierta medida institucional). De igual modo, si se prescinde de las líneas opuestas a la hipotenusa (siesta) solo quedaría una línea recta sin contexto, dejando de ser una unidad simbólica compleja.

Aquí es importante destacar lo que se denomina contexto de enunciación. El contexto, según Lyons (1989), es un constructo teórico que el lingüista abstrae de la situación real y así establece como contextuales todos los factores que, en virtud de una influencia sobre los participantes en el evento lingüístico, se llega a determinar de manera sistemática una forma, una adecuación y un significado de los enunciados.

Tampoco escapa a estas ideas el hecho de que el usuario de la lengua debe poseer un adecuado grado de competencia comunicativa. A este respecto, según Lyons (1989), la competencia comunicativa es un conocimiento y una capacidad de una persona para utilizar todos los sistemas semióticos que están disponibles en su entorno, en una comunidad sociocultural dada. La competencia lingüística o conocimiento cognitivo (y gramatical) es una parte de la competencia comunicativa.

Entonces, las palabras y las frases se originan en contextos en que la competencia comunicativa de los usuarios de la lengua discurre por factores de conocimiento de la situación, como oficios, estados, tiempo, espacio, capacidad de categorizar su mundo, etc.

\footnotetext{
${ }^{9}$ La primera hora correspondía a la salida del sol, el amanecer; por lo tanto, el almuerzo correspondía a la hora sexta.
} 


\section{Conclusiones}

Se puede concluir considerando los aspectos cognitivos como elementos de suma importancia para la comprensión de los pares etimológicos y que, en vista de la alomorfía, incomprensible para muchos no entendidos, se hace necesario el establecimiento de pautas cognitivas en la elaboración de diccionarios. La alternancia de morfos se hace recurrente en el vocabulario del español en sus correspondencias de especialización de la lengua a través de cultismos y tecnicismos, y correspondencias de uso particular desde la veta popular y evolutiva del léxico no especializado, tal como lo anota Varela (2005), y que se manifiesta en la enorme cantidad del léxico por préstamos de lenguas como el inglés.

Dos vetas del léxico español discurren entre los usuarios de este, marcándose una separación en los órdenes social, cultural e histórico. En estos órdenes, se reflejan los medios de transmisión de las palabras del latín al español como un continuum, según Dworkin (2012); constituyendo factores extralinguí́sticos que toman relevancia para el correcto análisis de los dobletes etimológicos.

Queda por establecer el presupuesto de que los dobletes etimológicos se configuran de manera distinta entre el diccionario y el thesaurum, según Halliday y Yallop (2007). En este, las entradas léxicas son de orden conceptual, unificadas por unidades semánticas o cognitivas, en el cual la información se programa dentro de valores enciclopédicos; mientras que en el diccionario, las entradas léxicas constituyen unidades independientes, descomponiendo la idea conceptual y reafirmando el problema conceptual-fonético.

\section{Bibliografía}

Aitchison, Jean. (1987). Words in the Mind. An Introduction to Mental Lexicon. Massachussets: Cambridge.

Bybee, Joan. (1985). Morphology: A Study of the Relation between Meaning and Form. Amsterdam: John Benjamins Publishing Company.

Cuenca, M. J. y Hilferty, J. (2007). Introducción a la lingüística cognitiva. Barcelona: Editorial Ariel. 
Dworkin, Steven. (2012). A History of the Spanish Lexicon. Oxford: Oxford University Press.

Gleitman, Lila y Landau, Barbara. (1996). Th e Acquisition of the Lexicon. Massachussets: MIT.

Halliday, M. A. K. y Yallop, Colin. (2007). Lexicology. Londres: Continuum.

Herman, Jozsef. (2001). El latín vulgar. Barcelona: Editorial Ariel.

Lara, Luis F. (2006). Curso de lexicología. México: El Colegio de México.

Lyons, John. (1989). Semántica. Barcelona: Editorial Teide.

Lyons, John. (1995). Semántica Lingüística: una introducción. Barcelona: Paidós.

Mattews, P.H. (1980). Morfología: Introducción a la teoría de la estructura de la palabra. Madrid: Paraninfo.

Moure, Teresa. (1996). La alternativa no-discreta en lingüística. Santiago de Compostela: Servicio de Publicaciones de la Universidad de Compostela.

Pino Méndez, E. (2009). Etimologias greco-latinas. Veracruz: Secretaría de Educación de Veracruz, México.

Panther, Klaus-Uwe y Radden, Günter. (2011). Motivation in Grammar and the Lexicon

Amsterdam: John Benjamins Publishing Company.

Quirós, M. A. (2000). El latín y las lenguas romances. San José: Editorial de la Universidad de Costa Rica.

Quirós, M. A. (2004). Latín hablado y latín clásico. San José: Editorial de la Universidad de Costa Rica.

Väänänen, V. (1975). Introducción al Latín Vulgar. Madrid: Editorial Gredos.

Varela Ortega, S. (2005). Morfología léxica: la formación de palabras. Madrid: Editorial Gredos. 